TPJU-7-2006

\title{
Comparison of the non-uniform chiral and 2SC phases at finite temperatures and densities
}

\author{
Mariusz Sadzikowski \\ Smoluchowski Institute of Physics, Jagellonian University, \\ Reymonta 4, 30-059 Kraków, Poland
}

\begin{abstract}
We study the phase diagram of the strongly interacting matter at finite temperatures and densities including 2SC, uniform chiral and non-uniform chiral phases within the Nambu - Jona-Lasinio model in the mean field approximation.
\end{abstract}

PACS numbers: 12.39.Fe;21.65.+f;12.38.Mh;64.70.-p 


\section{INTRODUCTION}

During the last decade a great effort has been made to understand the structure of matter at finite temperature and density [1]. As a generally accepted hypothesis the strongly interacting matter at high baryon density is in a color superconducting state. Although the first attempts in this direction had been made already long ago [2] only recently the subject achieved a new boost [3]. The simple observation was made that the quark-quark interaction at moderate densities should be of the same order as quark-antiquark interaction. This leads in turn to the sizable value of the superconducting gap $\Delta \sim O(100) \mathrm{MeV}$. What is more interesting it was proved that because of the non-abelian structure of the underlying fields in QCD, the superconduction gap grows with the chemical potential at very high density [4].

However, the main interest is concentrated at moderate densities possible to exist in the core of the compact stars. Using the selfconsistent calculation in the three flavor Nambu - Jona-Lasinio model the phase diagram of matter was elaborated under the reasonable

assumptions [5]. It occurs that depending on the value of the diquark coupling constant the 2SC (or g2SC) phase occupies the large region of the phase diagram at moderate densities and temperatures. In the construction of the phase diagram the superconducting phases are usually compared to the uniform chiral phase. However, there are also other possibilities.

It was shown that the chiral phase can develop the wave in the Lorentz scalar - pseudoscalar channels which has a lower energy than the uniform state [6]. This effect is well known in the low dimensional models (eg. 7]). Nevertheless, it can be argued that the phenomenon of the chiral wave might also appear in the $3+1$ dimensional QCD. The Nambu realization of the chiral symmetry breaking [8] is based on the idea borrowed from the BCS theory of superconductivity. The effective contact four-fermion interaction between quarks, described by the coupling constant $G$, is assumed to be attractive in the scalar, isoscalar channel $(G>0)$. It is then energetically favorable for the system to create the quarkantiquark pairs which built the Lorentz scalar chiral condensate. However QCD is invariant under $S U(2)_{V} \times S U(2)_{A} \times U(1)$ symmetry. As a result the effective quark interaction also contains pseudoscalar, isovector channel with the same coupling constant $G$. If the chiral wave develops in both channels then, as one can show, the wave vector acts effectively as a constant magnetic field which couples to the spin of the quarks. Then, in the analogy 
to the Zeeman effect, the quark energy levels split. The half of the energy branches lower their energy in compare to the uniform case, which depends on the quark spin projection on the wave vector axis. At finite density the quarks built the Fermi ladder, first filling the branches of the lower energy. This energy gain competes with the energy price due to the field gradients. There is a question of the detail calculations which contribution is more important. It was shown within the framework of the Nambu - Jona-Lasinio models or linear

sigma models [6, 9] that there is a wave vector range in which the energy gain overcome the price. The above scenario shares similarity with the Overhauser effect [10] which was already pointed out in the paper [11].

As one can see it is an important task to compare 2SC phases with non-uniform rather than uniform chiral phase. As a first attempt the 2SC phase was compared to the nonuniform chiral phase at zero temperature [12]. In this paper the calculation was extended to non-zero temperature. As a result the phase diagram in $T-\mu$ plane of the two flavor Nambu - Jona-Lasinio model including 2SC, uniform chiral and non-uniform chiral phases was calculated within the mean-field approximation.

\section{MEAN FIELD AND NAMBU-GORKOV FORMALISM}

We consider the NJL model at finite temperature:

$$
H=\int_{x}\left\{\bar{\psi}\left(i \gamma^{\nu} \partial_{\nu}+\mu \gamma_{0}\right) \psi+G\left[(\bar{\psi} \psi)^{2}+\left(\bar{\psi} i \gamma_{5} \vec{\tau} \psi\right)^{2}\right]+G^{\prime}\left(\bar{\psi}_{c} i \gamma_{5} \tau_{2} \lambda^{A} \psi\right)\left(\bar{\psi} i \gamma_{5} \tau_{2} \lambda^{A} \psi_{c}\right)\right\}
$$

where $\psi$ is the quark field, $\psi_{c}=C \bar{\psi}^{T}$ the conjugate field and $\mu$ is the quark chemical potential. The color, flavor and spinor indices are suppressed. The vector $\vec{\tau}$ is the isospin vector of Pauli matrices and $\lambda^{A}, A=2,5,7$ are three color antisymmetric $S U(3)$ group generators. The integration $\int_{x}=\int_{0}^{\beta} d \tau \int d^{3} x$, where $\beta$ is the inverse temperature and derivative operator $\partial_{\nu}=\left(i \partial_{\tau}, \vec{\nabla}\right)$. The coupling constant $G$ describes the interaction in the isospin singlet, Lorentz scalar and the isospin triplet, Lorentz pseudoscalar, quark-antiquark channels whereas $G^{\prime}$ describes the interaction in the color $\overline{\mathbf{3}}$, flavor singlet, Lorentz scalar diquark channel. Both couplings are treated as independent. In the momentum space a three momentum cut-off $\Lambda$ is introduced which finally defines the model.

Using the Hubbard-Stratonovich transformation one can perform partial bosonization of 
the model which leads to the standard expression

$$
\begin{array}{r}
H=\frac{1}{2} \int_{x}\left\{\bar{\psi}\left(i \gamma^{\nu} \partial_{\nu}+\mu \gamma_{0}+\sigma+i \gamma_{5} \vec{\pi} \cdot \vec{\tau}\right) \psi+\bar{\psi}_{c}\left(i \gamma^{\mu} \partial_{\mu}-\mu \gamma_{0}+\sigma+i \gamma_{5} \vec{\pi} \cdot \vec{\tau}^{T}\right) \psi_{c}\right. \\
\left.+G^{\prime}\left(\Delta_{A} \bar{\psi}_{c} i \gamma_{5} \tau_{2} \lambda^{A} \psi+\Delta_{A}^{*} \bar{\psi} i \gamma_{5} \tau_{2} \lambda^{A} \psi_{c}\right)-\frac{\sigma^{2}+\vec{\pi}^{2}}{2 G}-\frac{\Delta_{A} \Delta_{A}^{*}}{2 G^{\prime}}\right\} .
\end{array}
$$

We analyze the model in the mean field approximation within the following ansatz

$$
\begin{aligned}
& \sigma=-M \cos \vec{q} \cdot \vec{x} \\
& \pi_{a}=-M \delta_{a 3} \sin \vec{q} \cdot \vec{x} \\
& \Delta_{A}=\Delta \delta_{A 2}
\end{aligned}
$$

which takes into account three phases

- the homogenous chiral phase Ch when the wave vector $\vec{q}=0$, the gap parameter $\Delta=0$ and the constituent quark mass $M \neq 0$.

- The non-uniform chiral phase NCh when both the wave vector $\vec{q} \neq 0$ and constituent mass $M \neq 0$ whereas the gap parameter $\Delta=0$.

- The superconducting 2SC phase when $\vec{q}=0, M=0$ and $\Delta \neq 0$.

The chiral phases and 2SC phase could coexist with each other, as actually is the case for moderate densities, which was showed at $T=0$ [12]. One can also consider other condensates as for example the mixed states of $\pi^{+}, \pi^{-}$with the zero net charge [6, 9]. All conclusions given below which are based on the energy considerations does not change because the model treats all chiral fields at the same level.

The finite temperature analysis would be performed using Matsubara formalism in the Nambu-Gorkov representation. The partition function has a form

$$
Z=\int D \bar{\psi} D \psi D \bar{\psi}_{c} D \psi_{c} D \sigma D \vec{\pi} D \Delta_{A} D \Delta_{A}^{*} \exp H
$$

The mean field ansatz (3) is space dependent thus before integration over the fermionic fields one makes rotation $\psi^{\prime}=\sqrt{U} \psi$, where $U=\exp \left(i \gamma_{5} \tau_{3} \vec{q} \cdot \vec{x}\right)$. Then after introduction of the Nambu-Gorkov (NG) basis $\chi^{T}=\left(\psi^{\prime}, \psi_{c}^{\prime}\right)$ one arrives at the mean field action

$$
\begin{aligned}
& H_{M F}=\frac{1}{2} \int_{x}\left(\bar{\chi} S^{-1}(x, y) \chi-\frac{M^{2}}{2 G}-\frac{|\Delta|^{2}}{2 G^{\prime}}\right), \\
& S^{-1}(x, y)=\left[\begin{array}{cc}
i \gamma^{\nu}\left(\partial_{\nu}-\frac{1}{2} i \gamma_{5} \tau_{3} q_{\nu}\right)+\mu \gamma_{0}-M & i \gamma_{5} \tau_{2} \lambda_{2} \Delta \\
i \gamma_{5} \tau_{2} \lambda_{2} \Delta^{*} & i \gamma^{\nu}\left(\partial_{\nu}-\frac{1}{2} i \gamma_{5} \tau_{3} q_{\nu}\right)-\mu \gamma_{0}-M
\end{array}\right],
\end{aligned}
$$


where $q^{\nu}=(0, \vec{q})$. The bilinear form of $H_{M F}$ can be integrated over the fermionic fields with the mean field result

$$
Z_{M F}=\exp \left[-\int_{x}\left(\frac{M^{2}}{4 G}+\frac{|\Delta|^{2}}{4 G^{\prime}}\right)+\frac{1}{2} \ln \operatorname{det} S^{-1}\right] .
$$

Determinant of the inverse propagator is calculated in the momentum space

$$
S^{-1}(K)=\int_{K} S^{-1}(x, y) \exp (-i K(x-y)),
$$

where

$$
\int_{K}=T \sum_{n=-\infty}^{\infty} \int \frac{d^{3} k}{(2 \pi)^{3}}, \quad K^{\nu}=\left(-i \omega_{n}, \vec{k}\right), \quad \omega_{n}=(2 n+1) \pi T .
$$

The inverse propagator $S^{-1}(K)$ for any given $K$ is a $48 \times 48$ matrix in the color, flavor, Dirac and NG space. However, it decays into two block-diagonal sub-matrices: $16 \times 16$ matrix $S_{0}^{-1}$ and $32 \times 32$ matrix $S_{\Delta}^{-1}$. This simplification follows because the diquark interaction does not mixed the blue and red/green quarks. The calculation of these two determinants is straightforward although rather tedious and leads to the result

$$
\begin{aligned}
& \operatorname{det} S_{\Delta}^{-1}=\left[\prod_{i, k= \pm}\left(\epsilon_{i k}^{2}-K_{0}^{2}\right)\right]^{4}, \\
& \epsilon_{+, \pm}=\sqrt{\left(\mu+E_{ \pm}\right)^{2}+|\Delta|^{2}}, \quad \epsilon_{-, \pm}=\sqrt{\left(\mu-E_{ \pm}\right)^{2}+|\Delta|^{2}} \\
& E_{ \pm}=\sqrt{\vec{k}^{2}+M^{2}+\frac{\vec{q}^{2}}{4} \pm \sqrt{(\vec{q} \cdot \vec{k})^{2}+M^{2} \vec{q}^{2}}}
\end{aligned}
$$

where $\operatorname{det} S_{0}^{-1}=\operatorname{det}\left[S_{\Delta=0}^{-1}\right]$. After substitution of (9) into (6) one gets the grand potential $\Omega=-T \ln Z_{M F}$ in the form

$$
\begin{aligned}
\frac{\Omega}{V} & =V_{0}-4 T \int^{\Lambda} \frac{d^{3} k}{(2 \pi)^{3}} \sum_{i, k= \pm}\left(\ln \frac{1}{2}\left(1+\exp \frac{-\epsilon_{i k}}{T}\right)+\frac{1}{2} \ln \frac{1}{2}\left(1+\exp \frac{-\epsilon_{i k}^{0}}{T}\right)\right), \\
V_{0} & =\frac{M^{2}}{4 G}+\frac{|\Delta|^{2}}{4 G^{\prime}}-2 \sum_{i, k= \pm} \int^{\Lambda} \frac{d^{3} k}{(2 \pi)^{3}}\left(\epsilon_{i k}+E_{i}\right)+2 \sum_{i= \pm} \int_{E_{i} \leq \mu} \frac{d^{3} k}{(2 \pi)^{3}}\left(E_{i}-\mu\right), \\
\epsilon_{i k}^{0} & =\epsilon_{i k}(\Delta=0) .
\end{aligned}
$$

In the limit of zero temperature the second term of the potential $\Omega$ vanishes. It means that the term $V_{0}$ describes the zero temperature contribution. This term can be rewritten in the form useful for further calculation

$$
\begin{array}{r}
V_{0}=\frac{M^{2}}{4 G}+\frac{|\Delta|^{2}}{4 G^{\prime}}+\frac{M^{2} F_{\pi}^{2} \vec{q}^{2}}{2 M_{0}^{2}}-12 \int^{\Lambda} \frac{d^{3} k}{(2 \pi)^{3}} E_{0} \\
-2 \sum_{i= \pm}\left[\int^{\Lambda} \frac{d^{3} k}{(2 \pi)^{3}}\left(\sum_{k= \pm} \epsilon_{i k}-2 E_{i}\right)-\int_{E_{i} \leq \mu} \frac{d^{3} k}{(2 \pi)^{3}}\left(E_{i}-\mu\right)\right]
\end{array}
$$



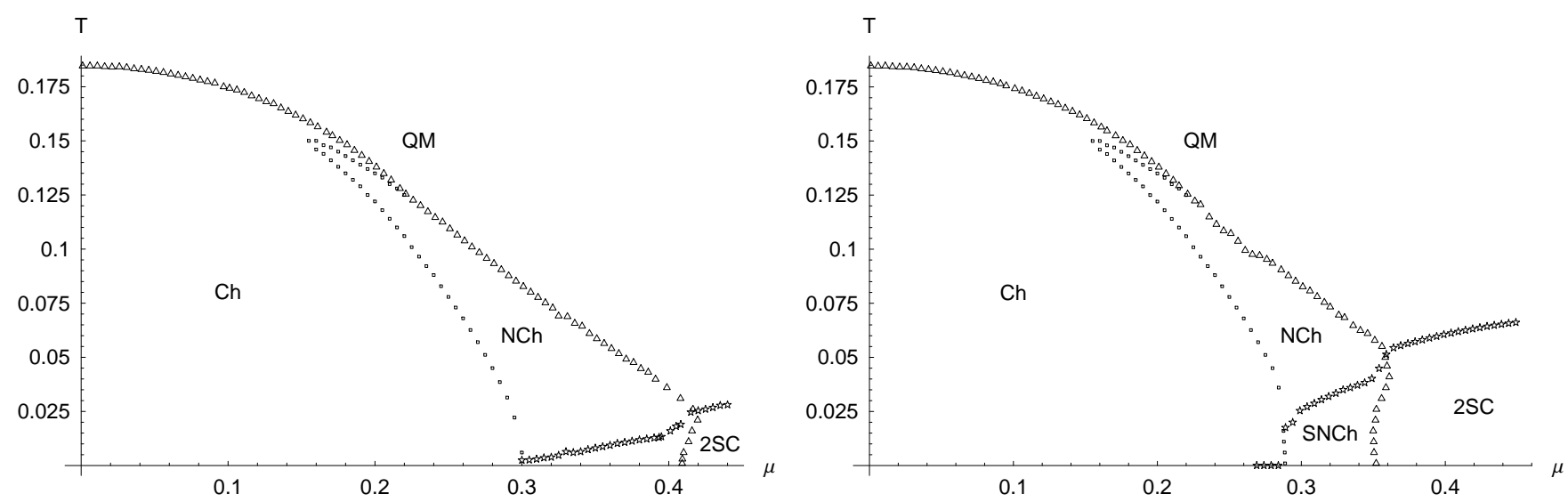

FIG. 1: The phase diagram in the $T-\mu$ plain in $\mathrm{GeV}$ for the coupling constants $G^{\prime}=G / 2$ (left panel) and $G^{\prime}=3 G / 4$ (right panel).

where the expansion in the power of the wave vector $\vec{q}$ was used as described in [12] and $M_{0}=0.301 \mathrm{GeV}$ is a constituent quark mass at zero density. The quantity $E_{0}=E_{i}(\vec{q}=0)$ and $F_{\pi}=93 \mathrm{MeV}$ is a pion decay constant.

\section{RESULTS}

The phase diagram describes the global minima of the potential $\Omega$ as a function of temperature $T$ and chemical potential $\mu$. Further constraints can be also imposed on the system as color and charge neutrality. We do not include them in this paper because its influence on the competition between chiral and 2SC phases is not quantitatively substantial. This problem would be discussed at the end of this section. The thermodynamic potential $\Omega=V_{0}+V_{T}$ is minimized with respect to $M, \vec{q}$ and $\Delta$. The zero temperature contribution $V_{0}$ is given by equation (11) and $V_{T}$ is the finite temperature part of expression (10) (second term of $\Omega$ ). The model parameters can be fitted to the values of the chiral condensate and the pion mass which give $G=5.01 \mathrm{GeV}^{-2}$ and the cut-off $\Lambda=0.65 \mathrm{GeV}$ [13]. The coupling constant $G^{\prime}$ was treated as independent from $G$. The minima were found using standard Nelder-Mead Simplex algorithm. The calculation was performed starting from three different points related to three different phases. The global minimum was that of the lowest value of the thermodynamic potential.

In the Fig. 1 the phase diagram for $G^{\prime}=G / 2$ (left panel) and $G^{\prime}=3 G / 4$ (right panel) are described. There are five different phases 
- the quark matter phase (QM) with $M=0, \vec{q}=0, \Delta=0$.

- The chiral phase (Ch) $M \neq 0 \vec{q}=0, \Delta=0$.

- The non-uniform chiral phase (NCh) $M \neq 0, \vec{q} \neq 0, \Delta=0$.

- The mixed phase of color superconducting and non-uniform chiral phases (SNCh) $M \neq 0, \vec{q} \neq 0, \Delta \neq 0$.

- The color supercoducting phase (2SC) $M=0, \vec{q}=0, \Delta \neq 0$.

Each of these phases differ by the order parameter and can be easily distinguished from each other. The phase transition lines between $\mathrm{Ch} / \mathrm{NCh}, \mathrm{QM} / \mathrm{NCh}, \mathrm{Ch} / \mathrm{SNCh}$ and $2 \mathrm{SC} / \mathrm{SNCh}$ are first order. This is connected to the fact that the entrance to the region of non-uniform condensate is associated with the jump in the value of the wave vector. This discontinues change entails the discontinues changes in the mass and gap parameters (see Fig. 2). The $\mathrm{QM} / \mathrm{Ch}, \mathrm{QM} / 2 \mathrm{SC}$ and $\mathrm{NCh} / \mathrm{SNCh}$ lines are second order phase transitions. This is nicely seen on the right panel of the Fig.2 which depicts the values of order parameters at the temperature $T=0.035 \mathrm{GeV}$ as a function of the chemical potential. First there is a 1st order phase transition from $\mathrm{Ch}$ into the $\mathrm{NCh}$ phase around $\mu=0.29 \mathrm{GeV}$ which is followed by the continuous phase transition into $\mathrm{SNCh}$ phase around $\mu=0.33 \mathrm{GeV}$ (rise of the gap parameter $\Delta$ ). Eventually there is the 1st order transition into the 2SC phase at $\mu=0.35 \mathrm{GeV}$, where both mass and the wave vector parameters vanish. The change of
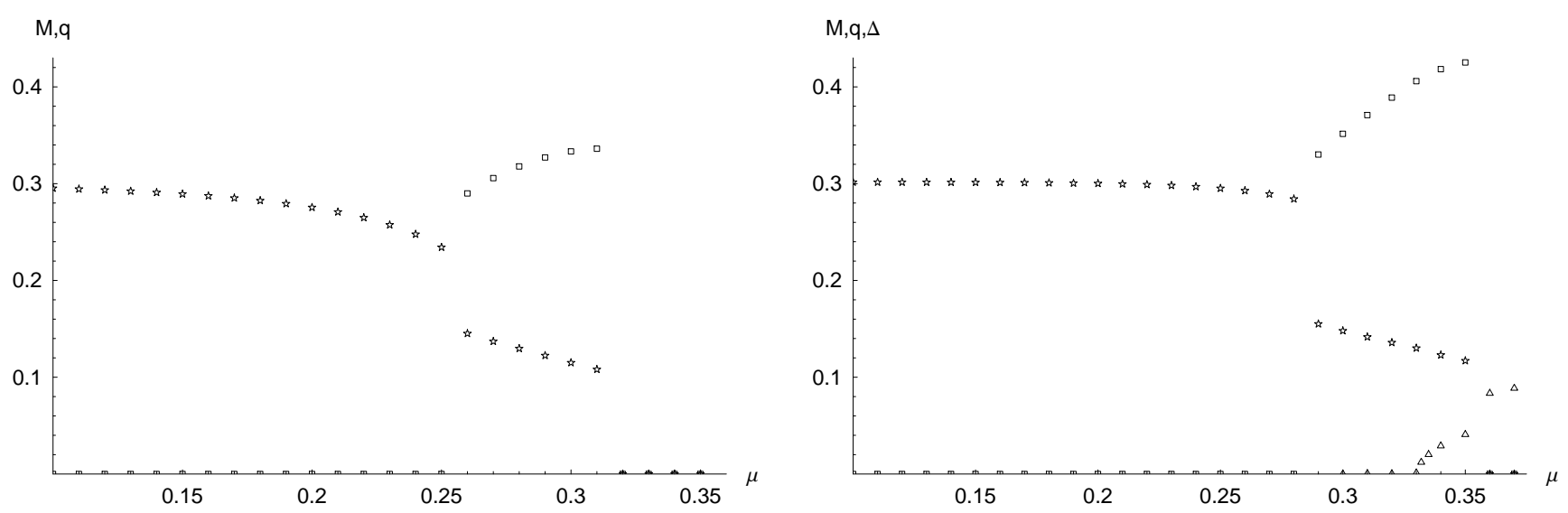

FIG. 2: The value of the mass $M$ (stars), wave vector $q$ (boxes) and the gap parameter $\Delta$ (triangles) for $T=0.075 \mathrm{GeV}$ (left panel) and $T=0.035 \mathrm{GeV}$ (right panel) for coupling constants $G^{\prime}=3 G / 4$. 
the character of the QM/Ch transition line from the second to the first order takes place in the merge point around $(\mu, T)=(0.221,0.125) \mathrm{GeV}$ where QM, Ch and NCh phases meets with each other.

The details of the phase diagram depends on the strength of the diquark coupling constant. The lower value of $G^{\prime}$ the smaller part of the diagram is covered with the pure 2SC phase. In the Fig. 1 it is seen that at low temperature the SNCh/2SC phase boundary starts at $\mu \approx 0.409 \mathrm{MeV}$ for $G^{\prime}=G / 2$ in compare to $\mu \approx 0.35 \mathrm{MeV}$ for $G^{\prime}=3 G / 4$. However when temperature grows both phase diagrams start to resemble each other (also quantitatively). Such a behavior is expected because the color superconductivity is rather low temperature phenomenon and does not influence the phase structure at higher temperature. In particular the region of $\mathrm{NCh}$ field is almost independent of the value of $G^{\prime}$ coupling constant. The only difference is that SNCh phase extends to the wider region. Also the melting temperature of the 2SC phase is larger at the right panel compare to the left one. This follows from the fact that the gap parameter takes larger values when $G^{\prime}=3 G / 4$.

The phase diagram at higher temperature $(T \approx 120 \mathrm{MeV})$ has to be treated with caution because of the corrections from the pion loops. In particular the characteristic insert of the NCh phase (the horn going to the left from the merge point of NCh/QM lines) possesses the wave vector of a small value $|\vec{q}| \ll \pi T$ thus the temperature fluctuations rather destroyed that part of the phase. Moreover the details of the phase diagram at higher temperature certainly depends on the regularization procedure, as one can conclude comparing Fig. 1 with similar from the paper [9]. The difference between these two approaches comes from the different way of the regularization of the $V_{T}$ part of the thermodynamic potential. Nevertheless at the lower temperature the results are robust within the expected accuracy of the NJL model.

Finally one has to remember that 2SC phase describes the colorful strongly interacting matter. The color charge neutrality condition can be easily accommodated through the additional color chemical potential [14]. The 2SC phase is suppressed under this condition thus as a result the $(\mathrm{S}) \mathrm{NCh}$ phases extend to the larger region of the diagram. However the change in the phase diagram structure is not substantial. At zero temperature it differs only in details at the few per cent level at most [15].

The influence of the beta equilibrium condition on the superconducting phases can result in the change of the phase structure of the color superconductor [16]. Then one needs rather 
to compare the non-uniform chiral phase with the LOFF superconducting phase. However, this task certainly requires different study and is outside the scope of the present paper.

\section{CONCLUSIONS AND FUTURE PROSPECTS}

In this paper the non-uniform chiral condensate NCh was compared to the uniform 2SC phase (3) at finite densities and temperatures. It was shown that the NCh phase exists in the temperature range between $20-100 \mathrm{MeV}$ above chemical potential $\mu \sim 250 \mathrm{MeV}$. It also coexists with 2SC phase at lower temperatures. The range of the phase coexistence depends on the relative strength of the chiral and diquark couplings. For $G^{\prime}=G / 2$ the density window open at $\mu \approx 0.3 \mathrm{GeV}$ and lasts for about $100 \mathrm{MeV}$ whereas for $G^{\prime}=3 G / 4$ it shrinks to about $50 \mathrm{MeV}$. The detailed phase diagram can be found in the Fig. 1 . It is worth to remind that $\mathrm{NCh}$ phase can have an important influence on the neutron star structure and also can be a source of the strong magnetic field [6, 17]. Then, the mixed state SNCh can have very unusual features as composed of NCh and 2SC phases. This interesting expectation remains to be revealed in some future work.

The light quarks are assumed to be massless in the model. The introduction of the nonzero mass leads to the tilt in the chiral wave as was shown in the low dimensional model 7]. There is not known exact solution to this problem in $3+1$ dimension. However, the effect has to be proportional to the current quark masses, which are the smallest energy scales in the system and should not have an important influence on energetic considerations. Nevertheless, the detailed analysis remains as an open question.

Another important problem is the comparison of the NCh phase and the color superconducting LOFF phase. This last phase appears naturally under the condition that matter is at beta equilibrium in the interior of the compact stars [18]. It is also consider as a remedy for the chromomagnetic instabilities in two flavor color superconductor [19]. At the first step one can try the simplified version of the problem when only two wave vectors for chiral and superconducting phase are assumed. The more general ansatz would be more ambitious, however, much harder to solve. Nevertheless the full analysis of the ground state at high baryon densities would require the study of the non-uniform phases of the chiral as well as diquark condensates. 


\section{Acknowledgments}

I would like to thank Piotr Bialas and Jan Kotanski for useful discussions. The research was supported by the MEiN grant 1P03B 04529 (2005-2008).

[1] M. Buballa, Phys. Rept. 407 (2005) 205; S. Reddy Acta Phys. Polon. B33 (2002) 4101.

[2] D. Bailin and A. Love, Nucl. Phys. B190[FS3] (1981) 175; B205[FS5] (1982) 119; Phys. Rep. 107 (1984) 325.

[3] M. Alford, K. Rajagopal and F. Wilczek, Phys. Lett. B422 (1998) 247; R. Rapp, T. Schafer and E. V. Shuryak, Phys. Rev. Lett. 81 (1998) 53.

[4] D. T. Son, Phys. Rev. D59 (1999) 094019.

[5] S. B. Ruster et al., Phys. Rev. D72 (2005) 034004; D. Blaschke et al., Phys. Rev. D72 (2005) 065020 .

[6] F. Dautry, E. M. Nyman, Nucl. Phys. A319 (1979) 323; T. Tatsumi, Prog. Theor. Phys. 63 (1980) 1252; M. Kutschera, W. Broniowski and A. Kotlorz, Nucl. Phys. A516 (1990) 566;

[7] V. Schön and M. Thies, in At the frontier of particle physics: HandBook of QCD, Boris Ioffe Festschrift, edited by M. Shifman, Vol. 3, Chap. 33, (2001), 1945.

[8] Y. Nambu and G. Jona-Lasinio, Phys. Rev. 122 (1961) 345; 124 (1961) 246.

[9] M. Sadzikowski and W. Broniowski, Phys. Lett. B488 (2000) 63.

[10] A. W. Overhauser, Phys. Rev. Lett. 4 (1960) 462; Phys. Rev. 128 (1962) 1437.

[11] E. Nakano and T. Tatsumi, Phys. Rev. D71 (2005) 114006.

[12] M. Sadzikowski, Mod. Phys. Lett. A16 (2001) 1129; Phys. Lett. B553 (2003) 45.

[13] S. P. Klevansky, Rev. Mod. Phys. 64 (1992) 64.

[14] M. Alford and K. Rajagopal, JHEP 0206 (2002) 031. M. Buballa and I. A. Shovkovy, Phys. Rev. D72 (2005) 097501.

[15] K. Domino, Msc. Thesis, Jagellonian University, 2006.

[16] M. Alford, J. Berges and K. Rajagopal, Nucl. Phys. B558 (1999) 219; T. Schäfer and F. Wilczek, Phys. Rev. D60 (1999) 074014.

[17] K. Takahashi, Prog. Theor. Phys. 108 (2002) 689.

[18] M. Alford and K. Rajagopal, hep-ph/0606157 (2006).

[19] I. Giannakis and H.-C. Ren, Nucl. Phys. B723 (2005) 255; I. Giannakis, D. Hou and H.-C. 
Ren, Phys. Lett. B631 (2005) 16. 